\title{
Fetal birthweight prediction with measured data by a temporal machine learning method
}

\author{
Jing Tao ${ }^{1,3}$, Zhenming Yuan ${ }^{2,4}$, Li Sun ${ }^{2,4}$, Kai Yu ${ }^{2,4}$ and Zhifen Zhang ${ }^{1,3^{*}}$ (D)
}

\begin{abstract}
Background: Birthweight is an important indicator during the fetal development process to protect the maternal and infant safety. However, birthweight is difficult to be directly measured, and is usually roughly estimated by the empirical formulas according to the experience of the doctors in clinical practice.

Methods: This study attempts to combine multiple electronic medical records with the B-ultrasonic examination of pregnant women to construct a hybrid birth weight predicting classifier based on long short-term memory (LSTM) networks. The clinical data were collected from 5,759 Chinese pregnant women who have given birth, with more than 57,000 obstetric electronic medical records. We evaluated the prediction by the mean relative error (MRE) and the accuracy rate of different machine learning classifiers at different predicting periods for first delivery and multiple deliveries. Additionally, we evaluated the classification accuracies of different classifiers respectively for the Small-forGestational-age (SGA), Large-for-Gestational-Age (LGA) and Appropriate-for-Gestational-Age (AGA) groups.
\end{abstract}

Results: The results show that the accuracy rate of the prediction model using Convolutional Neuron Networks (CNN), Random Forest (RF), Linear-Regression, Support Vector Regression (SVR), Back Propagation Neural Network(BPNN), and the proposed hybrid-LSTM at the 40th pregnancy week for first delivery were $0.498,0.662,0.670$, $0.680,0.705$ and 0.793 , respectively. Among the groups of less than 39th pregnancy week, the 39th pregnancy week and more than 40th week, the hybrid-LSTM model obtained the best accuracy and almost the least MRE compared with those of machine learning models. Not surprisingly, all the machine learning models performed better than the empirical formula. In the SGA, LGA and AGA group experiments, the average accuracy by the empirical formula, logistic regression (LR), BPNN, CNN, RF and Hybrid-LSTM were $0.780,0.855,0.890,0.906,0.916$ and 0.933 , respectively.

Conclusions: The results of this study are helpful for the birthweight prediction and development of guidelines for clinical delivery treatments. It is also useful for the implementation of a decision support system using the temporal machine learning prediction model, as it can assist the clinicians to make correct decisions during the obstetric examinations and remind pregnant women to manage their weight.

Keywords: Fetal birthweight prediction, Health data mining, Pregnant healthcare, Temporal data mining

\section{Background}

The fetal birthweight is an important indicator of the prognosis of the perinatal health. The correct prediction of the birthweight is undoubtedly of great significance

\footnotetext{
*Correspondence: zzfhangzhou@sina.com

${ }^{1}$ Department of Obstetrics and Gynecology, The Affiliated Hangzhou

People's Hospital of Nanjing Medical University, Hangzhou, China

Full list of author information is available at the end of the article
}

to determine the appropriate delivery method. It will be very important to reduce the occurrence of babies who are overweight or under gestational age and is also critical for both short-term and long-term health outcomes in neonates. Being born small for gestational age (SGA) is associated with seizures, respiratory distress, hypoglycemia, hyperbilirubinemia, polycythemia, thrombocytopenia and necrotizing enterocolitis. [1] On the other hand, the perinatal morbidity associated with large 
for gestational age (LGA) infants is related to a prolonged and complicated labor due to the physical size and includes birth injury, the need for operative vaginal delivery or caesarean section, asphyxia and meconium aspiration. Other postnatal problems that are commonly seen in LGA infants are hypoglycemia, hyperbilirubinemia, polycythemia and respiratory distress. On average, compared with neonates born appropriate for gestational age (AGA), SGA and LGA infants are more likely to need extra medical care during the delivery admission and readmission within two weeks of delivery. Therefore, estimating the birthweight during pregnancy helps to determine whether the fetal development is normal. Additionally, it has a guiding role in selecting the delivery mode during late pregnancy.

However, the birthweight cannot be directly measured before delivery and is usually roughly estimated according to the experience of the clinicians. Most empirical formulas come from applying regression analysis on the B-ultrasound measurement results of pregnant women. Shepard et al. [2] directly calculated the birthweight using parameters such as the biparietal diameter (BPD) and abdominal circumference (AC). Hadlock et al. [3] used the factors of head circumference (HC), abdominal circumference $(\mathrm{AC})$ and femur length (FL) to predict the birthweight via regression analysis. Zhu et al. [4] compared the accuracy of six empirical formulas using the uterine height and abdominal circumference and found the highest accuracy in the six empirical calculations to be $45.76 \%$, which does not meet the current clinical needs. Möstl et al. [5] considered that the traditional empirical formulas were based on the results of a singlepoint prediction, which made it easy to explain its significance but omitted the measurement of the uncertainty of the prediction intervals. Therefore, they proposed using the uncertainty to improve the robustness of the conditional linear transformation model in predicting the birthweight. Hong et al. [6] mentioned that the empirical formulas used in the clinical practice were mostly established for different areas. For example, there are racial differences among different ethnic groups in the measurement results. Therefore, appropriate adjustments need to be made to the empirical formulas' methods to adjust them for the specific circumstances. Furthermore, due to the differences in the maternal self-parameters and measurement methods, it is very difficult to establish a general empirical calculation, which leads to the low accuracy of the empirical prediction calculations [7].

Machine learning (ML) technologies have been recently used to predict the birthweight. Farmer et al. [8] firstly proposed an artificial neural network (ANN) for fetal body weight prediction using the results of the B-ultrasound to include the physical characteristics of pregnant women. They predicted the birthweight using a $\mathrm{BP}$ neural network (BPNN) according to the parameters of BPD, HC, AC, FL, amniotic fluid index time, birth, height and others. The results showed the BPNN to be better than the traditional regression analysis. Cheng et al. [9] proposed a clustering-based ANN model for birthweight prediction. Mohammadi et al. [10] used an ANN to predict the weight of twin fetuses. Feng $M$ [11] used an SVM and deep belief network (DBN) ML solution to improve the fetal weight estimation accuracy and to help the clinicians identify potential risks before delivery. Kuhle et al. [7] referred to the classification concept (SGA, AGA, LGA) of the birthweight with actual clinical values and compared and analyzed the prediction accuracy of the logarithmic weight classification (LR) and ML methods for different pregnancies. However, the abovementioned prediction models ignored the effect of timedependent factors.

In fact, the fetal birthweight is closely related to pregnant woman's pre-pregnancy weight and weight changes during pregnancy. Since the weight changes are time series data, this paper proposes a time series birthweight prediction model based on the long short-term memory (LSTM) networks. The model is trained on a large dataset of real maternal data, which is composed of the historical obstetric examinations and pregnancy outcomes of 5,759 pregnant women in China. The experimental results show that the hybrid prediction model improved the prediction accuracy by $14 \%$ compared with the traditional ML prediction method. Compared with the BPNN prediction model, the accuracy also improved by $6 \%$. In addition, we used this model to predict the birthweight classification (SGA, AGA and LGA), and the obtained results were better than those of ML methods.

\section{Methods}

Traditionally, the estimation of fetal weight in China is generally based on existing regression models that use multiple parameters established by foreign scholars. As a result, due to the individual differences in different populations, the use of these methods to estimate the weight of a fetus in China will result in large errors, especially for large especially for large or low-weight children. HybridLSTM model has obtained information from experience and mine complex concepts implicit in experience to make more efficient and reasonable decisions.

\section{The proposed birthweight prediction framework}

This study proposed to predict the birthweight using temporal prediction techniques. The block diagram of the prediction process is shown in Fig.1.

Overall, the input predictor set of temporal prediction model is comprised of pregnant women parameters, 


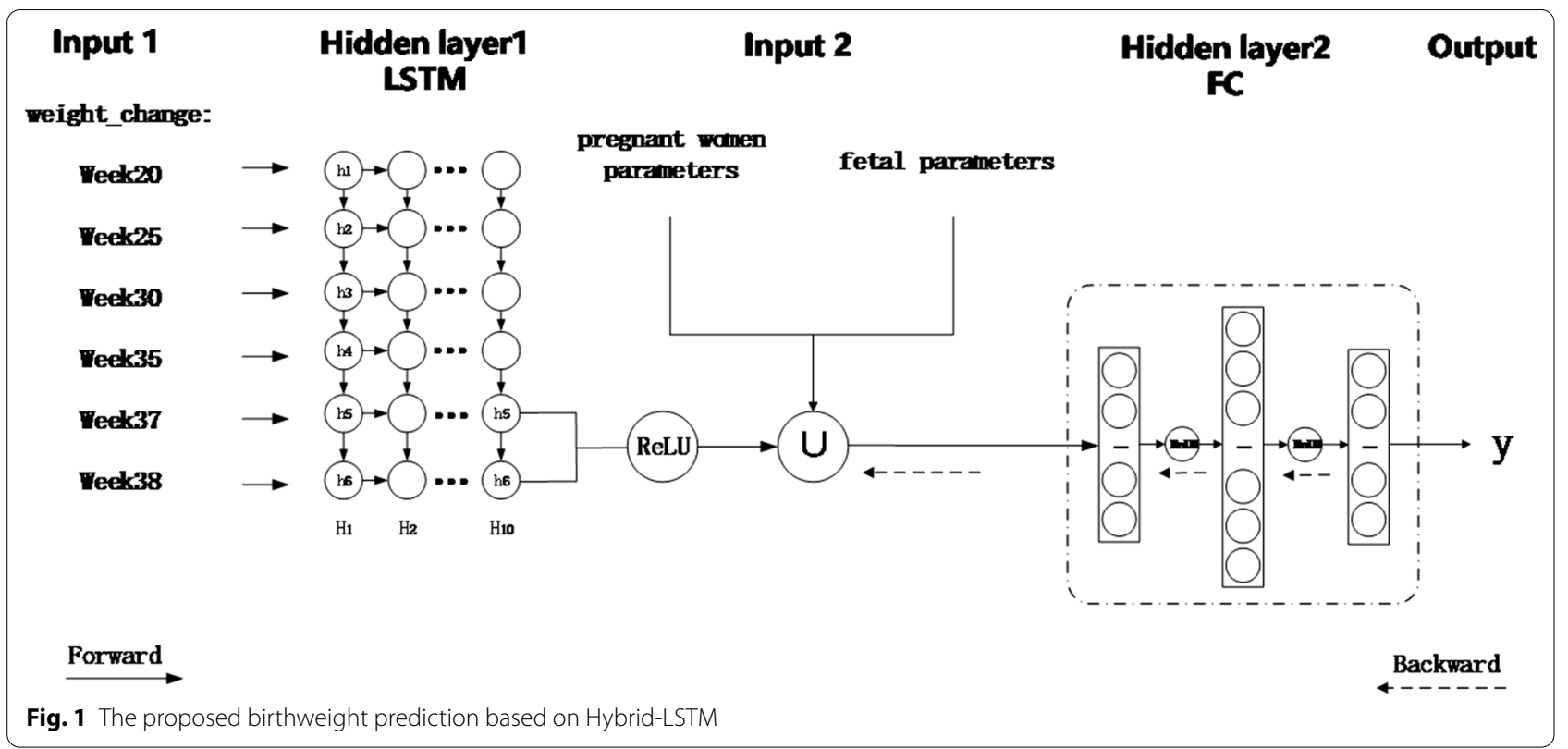

the fetal parameters and the weight change series. The relevant physiological fetal parameters are obtained via fetal ultrasonography, while the pregnant women parameters and the weight change series are obtained from the obstetric electronic medical records.

However, the frequency of antenatal examinations differs according to the different health conditions of pregnant women. In order to analyze the data, we need to standardize them in both time and magnitude dimensions. Therefore, data preprocessing is a key step toward improving the data quality. During the preprocessing, we processed the missing data and standardized the features of the raw data to form a continuously Hybrid-LSTM prediction model of the antenatal examination. The input layer is composed of two branches, two of which are combined as the input of the fully connected (FC) layers (Hidden layer 2). The activation function between the layers is the ReLU activation function, and then the output layer is a single neuron prediction. Finally, the predictive models quality was validated by standard concepts of accuracy as well as clinical usefulness.

\section{The predictors and the time series measured data}

The historical clinical data of pregnant women were saved in the electronic medical records (EMR) and the laboratory information management (LIS) systems. For this study, we used the ID card of pregnant women as the main index and extracted the inspection data from the beginning of pregnancy until the women gave birth. Then, the true birthweight and category (SGA, AGA, and LGA) of the newborn were used to form the output labels of the body weight predictors.
The model defines $\mathrm{Y}$ as a set of true birthweights and $\mathrm{X}$ as a model input parameter set. The input parameter set consists of 16 parameters, $\mathrm{X}=\{\{h, w, a, u h, a c p\},\{f l$, $a c f, b p d, h c, a f i\}$, \{weight_change $\}\}$, which correspond to the pregnant women parameters, the fetal parameters and the weight change series, respectively. Among them, the parameter set $\{h, w, a, u h, a c p\}$ represents the height, pre-pregnancy weight, pregnancy age, uterine height and abdominal circumference of pregnant women, respectively; $\{f, a c f, b p d, h c, a f i\}$ represents the femur length, abdominal circumference, biparietal diameter, head circumference and amniotic fluid index of the fetus. Table 1. List of parameters used in the prediction model. $\{\text { weight_change }\}_{t}$ represents the weight change series of pregnant women (the weight change refers to the value of the current weight minus the pre-pregnancy weight of pregnant women) at 6 different time points during pregnancy that are the 20th, 25th, 30th, 35th, 37th and 38th weeks. The denotations of the parameters are shown in Table 1.

As a time-series processing recurrent neural network (RNN), LSTM is suitable for processing and predicting the events with relatively long intervals and delays in the time series. Additionally, it can solve the gradient explosion problem when RNNs are used for long-term sequence predictions.

Each neuron of LSTM contains three control gates: the input gate, forget gate and output gate. The output at the previous time step enters the LSTM unit, and is then judged whether it is useful according to the cell. Only the useful information is kept, and the rest is forgotten at the forget gate.Equations (1) through (5) represent the 
Table 1 List of parameters used in the prediction model

\begin{tabular}{|c|c|c|c|c|}
\hline & Predictors & Notation & Predictors & Notation \\
\hline \multirow[t]{5}{*}{ Static predictors } & h & Height (cm) & w & Initial Weight (kg) \\
\hline & a & Age & uh & Uterine Height (cm) \\
\hline & acp & Abdominal Circumference of pregnant women (cm) & fl & Femur Length $(\mathrm{cm})$ \\
\hline & acf & Abdominal Circumference of fetal (cm) & bpd & Biparietal Diameter (cm) \\
\hline & hc & Head Circumference $(\mathrm{cm})$ & afi & Amniotic Fluid Index (cm) \\
\hline Time series predictors & weight_changet & Weight Change at 20th, 25th, 30th, 35th, 37th and 38th weeks) & & \\
\hline
\end{tabular}

parameter update process, where $\sigma$ represents the sigmoid function, $h_{t-1}$ represents the output of the LSTM at the previous time step, and $h_{t}$ represents the current output. The input gate, the forget gate and the output gate in the LSTM unit are defined as $i, f$, and $o$, respectively. $c_{t}$ is the state of the memory unit at the current time step, and Equation (4) represents the process of the state transition of the memory unit. The current state is calculated by the previous time step state, $c_{(t-1)}$ and the result of the forget gate and the input gate of the current time LSTM unit. In the data of this paper, the $\left\{\right.$ weight_change $\left.t_{t}\right\}$ represents the current input $x_{t}$. The structure of the LSTM unit is shown in Fig. 2.

$$
\begin{aligned}
& i_{t}=\sigma\left(\boldsymbol{W}_{x i} x_{t}+\boldsymbol{W}_{h i} h_{t-1}+b_{i}\right) \\
& f_{t}=\sigma\left(\boldsymbol{W}_{x f} x_{t}+\boldsymbol{W}_{h f} h_{t-1}+b_{f}\right)
\end{aligned}
$$

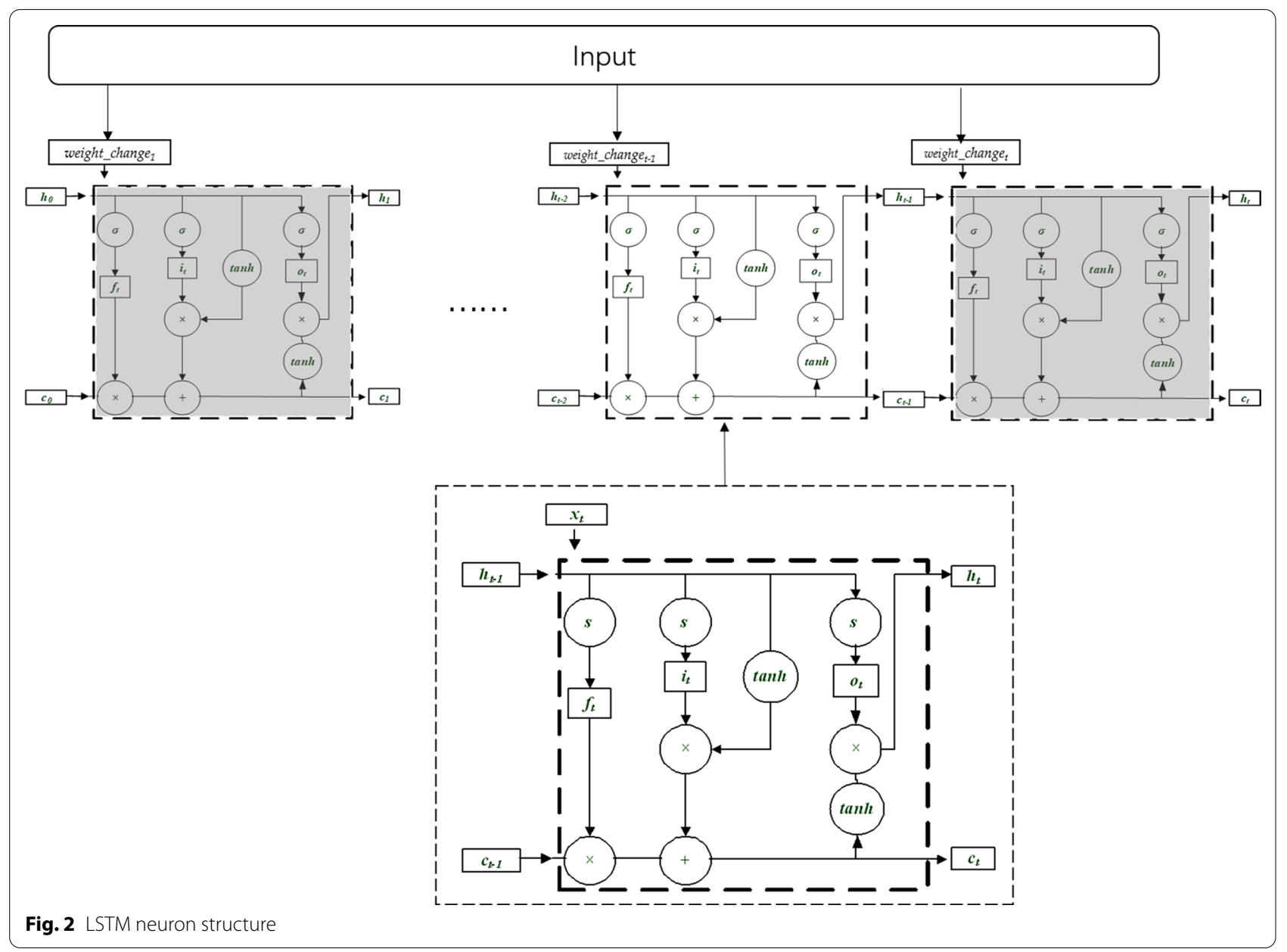




$$
\begin{aligned}
& o_{t}=\sigma\left(W_{x o} x_{t}+W_{h o} h_{t-1}+b_{o}\right) \\
& C_{t}=f_{t} c_{t-1}+i_{t} \tanh \left(W_{x c} x_{t}+W_{h c} h_{t-1}+b_{c}\right) \\
& h_{t}=o_{t} \tanh \left(c_{t}\right)
\end{aligned}
$$

\section{Hybrid-LSTM model \\ Data preprocessing}

Due to the variability of pregnancy check-ups date and the irregular inspection times, some loss will occur in the $\{\text { weight_change }\}_{t}$. This is a practical problem that exists in the forecasting model, which is based on historical inspection data. In this work, $\left\{\right.$ weight_change $\left.{ }_{t}\right\}$ with more than $20 \%$ missing values were excluded from further analysis. We used a regression method to fill in the missing weight values with a regression algorithm based on the existing weight data. Then, we screened the pregnant women with more than five antenatal examinations and computed their weight values at different times in their pregnancies (in weeks) using the regression method fitted by a quadratic fitting function. Given the data sequence $\left(x_{i}, y_{i}\right)$, assume $x_{i}$ to be the number of pregnancy weeks, $y_{i}$ to be the weight change at $x_{i}$ and $P(x)$ to be a quadratic fit function, the mean square error will then be calculated between the fitted function and the actual body weight sequence as follows:

$$
\sum_{i=1}^{m}\left(P\left(x_{i}\right)-y_{i}\right)^{2}
$$

The parameters of the quadratic fitting function are obtained by finding the minimum value of (1). In this study, we randomly selected 1000 sample data items for the fitting function experiment. The average relative error rate of the fitting result was $2.14 \%$. Using this method, we acquired a set of maternal body weight sequences $\left\{\right.$ weight_change $e_{t}$, and the missing value processing was completed.

After processing the missing value, a model input parameter set was obtained. However, since different physiological parameters have different units and orders of magnitude, and in order to eliminate the impact of different units and data levels on the prediction results of the model, the data were normalized before the input of the parameters into the network model to ensure that each parameter had the same order of magnitude [1214]. Standardization involved the calculation method shown in Equation (2), where $x$ represents the current eigenvalue, $x_{\min }$ and $x_{\max }$ represent the minimum and maximum values of the current eigenvalue, respectively, $y$ is the normalized eigenvalue and the normalized data range is $[-1,1]$.

$$
y=\frac{2\left(x-x_{\min }\right)}{x_{\max }-x_{\min }}-1
$$

\section{Structure of the hybrid-LSTM prediction model}

The structure of the hybrid-LSTM neural network is shown in Fig. 1. The input layer is composed of two branches, each branch is used to receive different types of the characteristic predictors. Input 1 is the weight change data, which consists of six different weight changes. This input is the input of the LSTM network (Hidden layer1), which has 6 input and 2 output dimensions. The number of layers that are superimposed on the LSTM network is 10. After obtaining the output of the LSTM layers, the multibranch input layer is used to divide the physiological parameters into different categories, then the model merges with the related pregnant women and fetal physiological parameters and uses them as the input for several FC layers (Hidden layer2). The final output layer is a single neuron prediction. The multibranch input layer is used to divide the physiological parameters into different categories.

Regarding the activation function between the layers, the activation function between the layers was the ReLU activation function, except for the hidden layer connected to the output layer at which we used the linear activation function.

According to the calculations at the LSTM unit in the previous section, the results of providing the weight change sequence as Input1 of the LSTM are the outputs $h_{5}$ and $h_{6}$ at time $H_{10}$, recorded as $x_{h 5}, x_{h 6}$, respectively. The fetal parameters and those of the pregnant women are recorded as $x_{\text {other }}$ Equation (8) represents the construction of the input parameters of the fully connected layer, and Equations (9) and (10) represent the input and output processing of the fully connected layer of each layer of the model, where $m$ is the number of current fully connected layer nodes (including the input layer), $w_{i j}$ is the weight between node $i$ and node $j, b_{j}$ is the threshold of node $j$ and the output value of each node is $y_{j}$, which is also the next $x$ of the fully connected layer; then, the final output of the model is the value of $y$ when $j$ is the last output layer node.

$$
\begin{aligned}
& x_{\text {init }}=\operatorname{ReLU}\left(x_{h 5}, x_{h 6}\right) \cup\left(x_{\text {other }}\right) \\
& S_{j}=\sum_{i=0}^{m-1} w_{i j} x_{i}+b_{j}
\end{aligned}
$$




$$
y_{j}=\operatorname{ReLU}\left(S_{j}\right)
$$

\section{Parameter settings of the hybrid-LSTM}

The Hidden layer2 includes 3 fully connected layers with the dimensions of $12 \times 20 \times 12 \times 1$. The number of neurons in the second layer is a relatively important factor that affects the convergence. Therefore, in this paper, we compare the effects of different dimensions by comparing different experiments while using the same data set and performing the same number of training epochs each time. The number of the second fully connected layer neurons is determined by comparing the mean square error (MSE), as shown in Equation (11).

$$
M S E=\frac{1}{m} \sum_{i=1}^{m}\left(y_{i}-\hat{y}_{i}\right)^{2}
$$

The best number of the second fully connected layer neurons is selected when the minimum MSE appears. The hybrid-LSTM neural network training has two stop conditions. The first condition is to reach the maximum number of epochs. The second condition is to have the error of 10 consecutive cycles less than the predetermined minimum error. Benefit from these two stop conditions, any unnecessary training time in the model can be reduced, and the overfitting phenomenon can be effectively prevented.

\section{Evaluation metrics for birthweight prediction}

In this study, we used two indicators to evaluate the performance of the proposed forecasting model. The first one is the mean relative error (MRE). This index can be used as a common evaluation standard in regression analysis, which well reflects the prediction accuracy and performance of the prediction model. It can be calculated as follows:

$$
M R E=\frac{1}{n} \sum_{i=1}^{n}\left|\frac{\hat{y}-y}{y}\right|
$$

where $y$ is the true value of the sample, $\hat{y}$ is the predicted value of the model and $\mathrm{n}$ is the total sample size.

The accuracy criterion is also considered together with MRE. Accuracy means that we will judge the prediction result to be correct when the error between the predicted and actual fetal body weight is within \pm 250 grams, which is acceptable for clinicians [15]. Accuracy can be calculated as Equation (13).

$$
\text { Accuracy }=\frac{1}{n} \sum_{i=1}^{n} \operatorname{bool}(|\hat{y}-y|<250)
$$

where $y$ is the true value of the sample, $\hat{y}$ is the predicted value of the model and $n$ is the total sample size

\section{The evaluation metric for birthweight classification}

The classification of the birthweight range has different standards in different countries and regions. This paper is based on the evaluation criteria for Eastern China. We identify a fetus with a birthweight of less than 2500 grams as small for gestational age (SGA), a fetus with a birthweight greater than 4000 grams as large for gestational age (LGA), and a fetus with a birthweight value between these two values as appropriate for gestational age (AGA).

SGA and LGA fetuses have potential risks of diseases; thus, it is practical to accurately predict the category of the fetus before delivery. We added a one-step classification operation for the model's predictions with the categories of SGA, AGA and LGA, we then labeled them with different numbers according to the specified weight range. Equation (14) is a description of the classification accuracy rate.

$$
\text { Category Accuracy }=\frac{1}{n} \sum_{i=1}^{n} \operatorname{bool}(\hat{y}=y)
$$

where $y$ is the true value of the sample, $\hat{y}$ is the predicted value of the model and $n$ is the total sample size.

\section{Results and discussion Dataset}

The dataset used in the paper comes from a hospital in Eastern China, which has a huge amount of data such as the maternal ultrasound records, birth check reports and imaging data. In this experiment, we selected 5,759 qualified samples from the obstetric electronic medical records between January 1, 2018 and October 1, 2018. The statistical information of pregnant women parameters, the fetal parameters and the weight change series is summarized in Table 2.

For the purpose of training the machine learning models and setting the parameters, we split the samples randomly into a training set and a test, with a $80 \%$ vs $20 \%$ ratio. Five-fold cross-validation was adopted in the evaluation experiments.

\section{Comparison of machine learning prediction models}

In order to more effectively verify the experimental results of this model, we compared the prediction results of the calculation formula, shown in Equation (15), with those of SVR and BPNN and compared the experimental results based on different births and delivery weeks.

The calculation formula uses the Zhuo's Formula, which has a relatively good prediction results compared with calculation formula [5]. In addition large sample 
Table 2 Summary statistics of pregnant women parameters, the fetal parameters and the weight change series in China, 2018.1-2018.10

\begin{tabular}{|c|c|c|c|c|c|}
\hline & Mean & Standard deviation & Minimum & Median & Maximum \\
\hline weight_change20w (kg) & 58.2 & 8.1 & 38.5 & 57.5 & 116.5 \\
\hline weight_change25w (kg) & 61.3 & 8.4 & 39.0 & 60.0 & 130.5 \\
\hline weight_change30w (kg) & 64.0 & 8.3 & 40.5 & 63.0 & 132.0 \\
\hline weight_change35w (kg) & 66.5 & 8.4 & 42.5 & 65.5 & 134.0 \\
\hline weight_change37w (kg) & 67.6 & 8.5 & 43.5 & 66.8 & 162.0 \\
\hline weight_change38w (kg) & 68.6 & 8.6 & 44.0 & 67.9 & 158.8 \\
\hline age & 29.3 & 4.2 & 18.0 & 29.0 & 46.0 \\
\hline height (cm) & 160.4 & 4.8 & 144.0 & 160.0 & 184.0 \\
\hline initial weight (kg) & 54.7 & 8.1 & 35.0 & 54.0 & 163.0 \\
\hline $\operatorname{acp}(\mathrm{cm})$ & 100.2 & 6.0 & 73.0 & 100.0 & 145.0 \\
\hline uh $(\mathrm{cm})$ & 34.4 & 1.9 & 26.0 & 34.0 & 73.0 \\
\hline $\mathrm{bpd}(\mathrm{cm})$ & 9.3 & 0.3 & 7.9 & 9.3 & 10.5 \\
\hline $\mathrm{hc}(\mathrm{cm})$ & 33.2 & 1.0 & 29.3 & 33.2 & 39.2 \\
\hline $\operatorname{acf}(\mathrm{cm})$ & 34.1 & 1.5 & 28.9 & 34.1 & 41.7 \\
\hline $\mathrm{fl}(\mathrm{cm})$ & 7.2 & 0.2 & 5.9 & 7.2 & 8.2 \\
\hline afi & 10.6 & 3.6 & 2.0 & 10.3 & 29.6 \\
\hline birthweight (g) & 3399.1 & 385.8 & 1520.0 & 3390.0 & 4950.0 \\
\hline parity & 0.4 & 0.5 & 0 & 0 & 3.0 \\
\hline delivery weeks & 39.4 & 0.9 & 37.0 & 39.0 & 44.0 \\
\hline
\end{tabular}

studies have proved that Zhuo's Formula has clinical significance without distinguishing LGA, SGA and AGA [4], as follows:

$$
G=u h * 100
$$

where $G$ is the predicted weight, and $u h$ is the uterine height.

The machine learning method that we used is the SVM regression (SVR), which has been widely applied in medical diagnostics with small sample sets, the BPNN is the standard 3-layer neural network, Linear regression(Linear- $\mathrm{R}$ ) is a statistical analysis method to determine the quantitative relationship among multiple variable, the $\mathrm{CNN}$ is a kind of feed forward neural networks with depth structure and convolution computation, and the RF is a decision tree algorithm based on bagging ensemble.

Based on the above-mentioned features in Table 3, we constructed these machine learning models to predict the birthweight. Using the optimal parameters for each model, the predictive models were corroborated via a validation set which was derived from the training dataset by 5 -fold cross-validation.

The corresponding MSE values after training the different network structures are shown in Table 4, when the number of neurons in the layer is 20 , the network performance is the best. As we continue to increase the number of hidden layer neurons, the network performance does not improve. Therefore, the number of the second FC layer neurons was determined to be 20.

The model's parameter settings for the dropout [16, 17] were used between the hidden layers to prevent the model from overfitting, and the dropout parameter was set to 0.2. Meanwhile, the batch size was set to 50 . The model used the mean square error (MSE) as the loss function with Adam [18] as the optimizer.

\section{Overall comparison results}

First, we compared the prediction effects of different methods based on all the data. The average values of the results after 5 -fold CV are shown in Table 5.

As shown in Table 5, Our study found that the hybridLSTM prediction model, outperformed Calculation formul, SVR, BPNN, Linear-R, CNN and RF, achieved average accuracy of $>0.79$, and the error of the hybridLSTM prediction model for the birthweight is controlled within $5.7 \%$, and the results of this method are far superior to several other prediction methods. This suggests that hybrid-LSTM prediction models have better generalization capabilities compared to other models for predicting the actual fetal body weight. Even though Linear-R and RF also has lower MRE, this might not be acceptable as lower accuracy means that these model might not perform well in AGA. The results of our study 
Table 3 Summary of parameter values in each model

\begin{tabular}{|c|c|c|c|}
\hline Models & Parameters & Values & Parameters Meaning \\
\hline \multirow[t]{5}{*}{ SVM } & kernel & linear & kernel function \\
\hline & C & 1.0 & regularization parameter \\
\hline & Cache_size & 200 & specify the size of the kernel cache \\
\hline & tol & 0.001 & tolerance for stopping criterion \\
\hline & gamma & scale & kernel coefficient \\
\hline \multirow[t]{6}{*}{ BPNN } & kernel initializer & uniform & kernel initializer function \\
\hline & activation 1 & relu & activation of hidden layer \\
\hline & activation2 & sigmoid & activation of output layer \\
\hline & optimizer & Adam & training optimization algorithm \\
\hline & epochs & 200 & number of times shown to the network \\
\hline & batch size & 128 & batch size \\
\hline \multirow[t]{3}{*}{ Linear-R } & fit_intercept & True & whether to calculate the intercept for this model \\
\hline & normalize & False & whether to standardize the data \\
\hline & copy_X & True & If True, $X$ will be copied \\
\hline \multirow[t]{11}{*}{ CNN } & Ir & 0.01 & learning rate \\
\hline & epochs & 100 & number of times shown to the network \\
\hline & optimizer & Adam & training optimization algorithm \\
\hline & Conv1_in_channels & 1 & number of channels in the input image \\
\hline & Conv1_outchannels & 10 & number of channels produced by the convolution \\
\hline & Conv1_kernel_size & 1 & size of the convolving kernel \\
\hline & Conv1_strid & 2 & stride of the convolution \\
\hline & Conv2_in_channels & 10 & number of channels in the input image \\
\hline & Conv2_outchannels & 20 & number of channels produced by the convolution \\
\hline & Conv2_kernel_size & 1 & size of the convolving kernel \\
\hline & Conv2_strid & 2 & stride of the convolution \\
\hline \multirow[t]{5}{*}{ RF } & n estimators & 200 & the number of trees in the forest \\
\hline & Min_samples_leaf & 1 & the minimum number of samples required to be at a leaf node \\
\hline & Min_samples_split & 2 & the minimum number of samples required to split an internal node \\
\hline & max depth & None & the maximum depth of the tree. \\
\hline & max features & 7 & the number of features to consider when looking for the best split \\
\hline
\end{tabular}

Table 4 MSE after the training of different network structures

\begin{tabular}{ll}
\hline Number of second FC layer neurons & MSE \\
\hline 12 & 0.0553 \\
14 & 0.0424 \\
16 & 0.0281 \\
18 & 0.0212 \\
20 & 0.0146 \\
22 & 0.0217 \\
25 & 0.0256 \\
30 & 0.0319 \\
\hline
\end{tabular}

indicate that hybrid-LSTM prediction model are well suited for the prediction of fetal weight within acceptable error range in most people.
Table 5 Prediction average results of different methods after 5-fold CV

\begin{tabular}{lll}
\hline Method & MRE (\%) & Accuracy (\%) \\
\hline Zhuo's Formula & $8.52 \pm 0.4$ & 58.7 \\
SVM-R & $7.33 \pm 0.4$ & 65.5 \\
BPNN & $6.36 \pm 0.4$ & 73.0 \\
Linear-R & $5.77 \pm 0.4$ & 70.4 \\
CNN & $9.21 \pm 0.4$ & 51.0 \\
RF & $5.86 \pm 0.4$ & 69.9 \\
Hybrid-LSTM & $5.65 \pm 0.4$ & 79.2 \\
\hline
\end{tabular}

\section{Performance comparison by delivery weeks}

In addition, to compare the effects of different delivery weeks and parities on the predictive effect, we divided the data into the first and multiple deliveries. On this 
basis, the data were divided into the groups of less than 39 weeks, 39 weeks, 40 weeks and more than 40 weeks of delivery.

As shown in Table 6, our proposed method achieved better prediction results than the other methods for different births and delivery weeks. However, regarding the data for multiple delivery women with more than 40 weeks of delivery, the prediction results were poor and were 10 percentage points lower than the average. The prediction was the best for the data of single-child pregnant women for less than 39 weeks, reaching approximately $85 \%$. This shows that the relationship between the birthweight and the parameters of pregnancy is more stable for first delivery women than for multiple delivery ones. More than 37 weeks of delivery is a medically recognized full-term fetus. The results of this experiment showed that the predicted birthweights are more accurate and reliable as the delivery time is more within the normal range.

\section{Comparion of birthweight classification results}

We compared our model with the Zhuo's Formula [5], the logistic regression (LR) method [7] and the BPNN, CNN and RF as shown in Table 7.

Table 7 shows that the model exhibited a good ability to predict the classification of the birthweight, which does not only reduce the risk of fetal diseases but can
Table 7 Prediction results of different methods

\begin{tabular}{ll}
\hline Method & Accuracy (\%) \\
\hline Formula & 78.0 \\
LR & 85.5 \\
BPNN & 89.0 \\
CNN & 90.6 \\
RF & 91.6 \\
Hybrid-LSTM & 93.3
\end{tabular}

also help the doctors to make appropriate clinical decisions and improve the pregnancy success rate.

\section{Conclusion}

In the paper, we proposed a hybrid birthweight prediction model based on LSTM, which establishes a continuous model of the parameters related to the pregnant women and fetal physical examination. The experimental results show that the proposed birthweight prediction model does not only increase the model convergence rate but also improves the birthweight prediction accuracy by $6 \%$. There is room for improvement in the deep neural network model to improve the accuracy and practicality of the model prediction. Additionally, this study explored the risk classification of different categories of the birthweight, and we obtained better accuracy than other methods, which can be useful for clinical applications.

Table 6 Prediction results for different periods

\begin{tabular}{|c|c|c|c|c|c|c|c|c|c|}
\hline \multirow{2}{*}{$\begin{array}{l}\text { Delivery } \\
\text { Evaluation }\end{array}$} & & \multicolumn{2}{|c|}{ Less than 39 weeks } & \multicolumn{2}{|c|}{39 weeks } & \multicolumn{2}{|c|}{40 weeks } & \multicolumn{2}{|c|}{$\begin{array}{l}\text { More than } 40 \\
\text { weeks }\end{array}$} \\
\hline & & MRE & Accuracy & MRE & Accuracy & MRE & Accuracy & MRE & Accuracy \\
\hline \multirow[t]{7}{*}{ First delivery } & Formula & 8.3 & 59.5 & 8.9 & 54.5 & 8.7 & 56.3 & 8.8 & 52.3 \\
\hline & SVM & 7.2 & 68.7 & 7.3 & 67.0 & 7.4 & 68.0 & 7.6 & 65.4 \\
\hline & BPNN & 6.2 & 75.0 & 6.7 & 72.0 & 6.5 & 70.5 & 6.2 & 71.2 \\
\hline & Linear-R & 6.0 & 70.1 & 5.8 & 68.4 & 6.1 & 67.0 & 5.8 & 69.2 \\
\hline & CNN & 7.3 & 63.6 & 8.8 & 51.6 & 9.5 & 49.8 & 9.8 & 51.4 \\
\hline & RF & 5.9 & 75.3 & 5.4 & 70.7 & 5.9 & 66.2 & 6.1 & 65.4 \\
\hline & Hybrid-LSTM & 5.11 & 84.8 & 6.0 & 76.7 & 5.7 & 79.3 & 5.7 & 81.0 \\
\hline \multirow[t]{7}{*}{ Multiple deliveries } & Formula & 7.9 & 61.0 & 8.6 & 56 & 8.1 & 60.5 & 9.2 & 51.5 \\
\hline & SVM & 6.9 & 70.3 & 7.2 & 62 & 7.6 & 67.0 & 7.6 & 63.0 \\
\hline & BPNN & 6.5 & 75.7 & 6.3 & 73 & 6.7 & 71.0 & 6.8 & 68.2 \\
\hline & Linear-R & 5.9 & 69.2 & 6.0 & 72.4 & 5.5 & 68.7 & 7.9 & 61.1 \\
\hline & CNN & 10.1 & 43.8 & 8.3 & 53.5 & 10.1 & 46.3 & 8.7 & 44.4 \\
\hline & $\mathrm{RF}$ & 7.4 & 61.5 & 5.8 & 70.6 & 6.5 & 67.2 & 7.2 & 66.7 \\
\hline & Hybrid-LSTM & 5.3 & 81.6 & 5.7 & 78.6 & 6.1 & 74.8 & 7.3 & 69.0 \\
\hline
\end{tabular}




\section{Abbreviations}

LSTM: Long short-term memory; MRE: Mean relative error; SGA: Small-forgestational-age; LGA: Large-for-gestational-age; AGA: Appropriate-forgestational-age; CNN: Convolutional neuron network; RF: Random forest; SVR: Support vector regression; LR: Logistic regression; BPD: Biparietal diameter; AC: Abdominal circumference; HC: Head circumference; FL: Femur length; ML: Machine learning; ANN: Artificial neural network; DBN: Deep belief network; FC: Fully connected; LIS: Laboratory information management; EMR: Electronic medical records; RNN: Recurrent neural network; MSE: Mean square error; CV: Cross-validation.

\section{Acknowledgements}

The authors would like to thank research assistants Peilin Li and Xuhong Zhu for their efforts in data collection and management. The authors would also like to thank all pregnant women and healthcare professionals who participated in the different stages of the development of the prediction algorithm.

\section{Authors' contributions}

Conceptualization, JT and ZZ; methodology, JT and ZY; software, $K Y$ and ZY; validation, JT and LS; formal analysis, $Z Y$; investigation, JT; data curation, $K Y$; writing-original draft preparation, JT; writing-review and editing, JT and ZY; visualization, ZY; supervision, ZZ; project administration, ZZ; funding acquisition, JT. All authors have read and agreed to the published version of the manuscript. All authors read and approved the final manuscript.

\section{Funding}

This research was funded by NSF of Zhejiang Province in China, grant number GF20F020063, which provide financial support $n$ the design of the study and collection, analysis, and interpretation of data and in writing the manuscript.

\section{Availability of data and materials}

The data that support the findings of this study are available from Hangzhou Women's Hospital, but restrictions apply to the availability of these data, which were used under license for the current study, and so are not publicly available. Data are however available from the authors upon reasonable request and with permission of Hangzhou Women's Hospital.

\section{Ethics approval and consent to participate}

This study is observational and presents no more than minimal risk of harm to subjects and involves no procedures for which written consent is normally required outside the research context. Hangzhou Women Hospital Research Ethics Board approved the waiver of informed consents (NO. 2017-001) for this study according to section 39 of Measures for the Ethical Review of Biomedical Research Involving Humans published by National Health Commission of the People's Republic of China (CLI.4.282697).

\section{Consent for publication}

Not applicable.

\section{Competing interests}

The authors declare that they have no competing interests.

\begin{abstract}
Author details
${ }^{1}$ Department of Obstetrics and Gynecology, The Affiliated Hangzhou People's Hospital of Nanjing Medical University, Hangzhou, China. ${ }^{2}$ Engineering Research Center of Mobile Health Management Ministry of Education, Hangzhou Normal University, Hangzhou, China. ${ }^{3}$ Department of Obstetrics and Gynecology, Hangzhou Women's Hospital, Hangzhou, China. ${ }^{4}$ Department of Research and Development, Hangzhou Hele Tech.Co, Hangzhou, China.
\end{abstract}

Received: 8 May 2020 Accepted: 6 January 2021

Published online: 25 January 2021

\section{References}

1. Yu Z, Han S, Zhu J, Sun X, Ji C, Guo C. Pre-pregnancy body mass index in relation to infant birth weight and offspring overweight/obesity: a systematic review and meta-analysis. PLOS ONE. 2013;8(4):61627.
2. Shepard M, Richards V, Berkowitz R, Warsof S, Hobbins J. An evaluation of two equations for predicting birthweight by ultrasound. Am J Obstetr Gyneco. 1982;142(1):47-544.

3. Hadlock F, Harrist R, Carpenter R, Deter R, Park S. Sonographic estimation of birthweight. The value of femur length in addition to head and abdomen measurements. Radiology. 1984;150(2):535-40.

4. Zhu T, Zhao X, Ai M, Ma R, Lei J, Liu J. Comparison of accuracy of six calculations for predicting fetal body weight. Chin Mater Child Health Care. 2016:(20):4179-81.

5. Möst L, Schmid M, Faschingbauer F, Torsten H. Predicting birth weight with conditionally linear transformation models. Chin Mater Child Health Care. 2016;25(6):2781-810.

6. Hong C, Ji Y. Comparison of common methods for birthweight prediction and clinical value. Chin Women Children Health Res. 2017:5:2781-810.

7. Kuhle S, Maguire B, Zhang H, Hamilton D, Allen A, Joseph K, Victoria M. Comparison of logistic regression with machine learning methods for the prediction of fetal growth abnormalities: a retrospective cohort study. BMC Pregn Childbirth. 2018;18(1):333.

8. Farmer R, Medearis A, Hirata G, Platt L. The use of a neural network for the ultrasonographic estimation of birthweight in the macrosomic fetus. Am J Obstetr Gynecol. 1992;166(5):1467-72.

9. Cheng Y, Hsia C, Chang F, Hou C, ChiU Y, Chung K. Zeolites and synthetic mechanisms. In: 6th World Congress of Biomechanics (WCB 2010), Singapore 2010;1514-1517

10. Mohammadi H, Nemati M, Allahmoradi Z, Forghani H, Sheikhani A. Ultrasound estimation of birthweight in twins by artificial neural network. J Biomed Sci Eng. 2011;4(1):46-50.

11. Feng $M$, Wan $L$, Li Z, Qing $L$, Qi X. Fetal weight estimation via ultrasound using machine learning. IEEE Access. 2019;7:87783-91.

12. Chen G, Fu K, Liang Z, Sema T, Li C, Idem R. The genetic algorithm based back propagation neural network for MMP prediction in $\mathrm{CO}_{2}$-EOR process. Fuel. 2014;126:202-12.

13. Rumelhart $D$, Hinton $G$, Williams R. Learning internal representations by error propagation. In: Parallel distributed processing: Explorations in the microstructures of cognition. Cambridge: MIT Press; 1987. p. 318-362.

14. Krizhevsky A, Sutskever I, Hinton G. Imagenet classification with deep convolutional neural networks. Commun ACM. 2017;60(6):84-90.

15. Lu Y, Fu X, Chen F, Wong K. Prediction of fetal weight at varying gestational age in the absence of ultrasound examination using ensemble learning. Artif Intell Med. 2020;102:101748.

16. Yang Q, Jiang J, Zhang G. Improvement of genetic algorithm optimization speed. Softw J. 2001:12(2):270-5.

17. Srivastava N, Hinton G, Krizhevsky A, Sutskever I, Salakhutdinov R. Dropout: A simple way to prevent neural networks from overfitting. J Mach Learn Res. 2014;15(1):1929-58.

18. Kingma DP, Ba J. Adam: A method for stochastic optimization. In: the 3rd International Conference for Learning Representations(ICLR), San Diego, 2015.

\section{Publisher's Note}

Springer Nature remains neutral with regard to jurisdictional claims in published maps and institutional affiliations.

Ready to submit your research? Choose BMC and benefit from

- fast, convenient online submission

- thorough peer review by experienced researchers in your field

- rapid publication on acceptance

- support for research data, including large and complex data types

- gold Open Access which fosters wider collaboration and increased citations

- maximum visibility for your research: over $100 \mathrm{M}$ website views per year

At BMC, research is always in progress.

Learn more biomedcentral.com/submissions 